\title{
Smart Motion Detection System using Raspberry Pi
}

\author{
Priya B. Patel \\ M.Tech Student \\ Dept. of Embedded \\ System \\ Sardar Vallabhbhai \\ Institute of Technology \\ Vasad-Gujarat \\ India
}

\author{
Viraj M. Choksi \\ Project Scientist \\ Bhaskaracharya \\ Institute for Space \\ Applications and \\ Geo-informatics \\ Gandhinagar-382007. \\ Gujarat, India
}

\author{
Swapna Jadhav \\ Assistant Professor \\ Dept. Of Electronics \\ and Communication \\ Sardar Vallabhbhai \\ Institute Of Techology \\ Vasad-Gujarat \\ India
}

\author{
M.B. Potdar, PhD \\ Bhaskaracharya \\ Institute for Space \\ Applications and \\ Geo-informatics \\ Gandhinagar-382007 \\ Gujarat, India
}

\begin{abstract}
In surveillance, CCTV camera is costly because of the use of computer. It reserves too much space for continues recording and also require manpower to detect the unauthorized Activity. But compared to the existing system Raspberry pi system is much cheaper with better resolution and low power consumption feature. Here pyroelectric infrared (PIR) sensors are used as a simple but powerful people presence triggers. This system is suitable for small personal area surveillance. i.e. personal office cabin, bank locker room, parking entrance. Whenever the motion is detected through PIR sensor inside the room the image is captured through camera and temporarily stored in the raspberry pi module. Internet of things based application can be used remotely to view the activity and get notifications when motion is detected. System works standalone without the PC once programmed. One android Application is used to get the notification on motion detection.
\end{abstract}

\section{Keywords}

Raspberry Pi, PIR sensor, Android Application

\section{INTRODUCTION}

Starting from small houses to huge industries, surveillance plays very vital role to fulfill our safety aspects as Burglary and theft have always been a problem. In big industries personal security means monitoring the people's changing information like activities, behavior for the purpose of protecting, managing and influencing confidential details. Surveillance means watching over from a distance by means of electronic equipment such as CCTV cameras ${ }^{[3]}$ but it is costly for normal residents to set up such kind of system and also it does not inform the user immediately when the burglary happens.

\subsection{Motivation}

The use of M2M (machine to machine) communication is an advantage over the traditional Data Acquisition System (DAS) as the monitoring and controlling can be done without human intervention ${ }^{[2]}$. As the system becomes fully automatic so the amount of error decreases and the efficiency of the system increases drastically.

\subsection{Advantages}

It offers privacy on both sides since it is being viewed by only one person. It is a simple circuit. The operating system used here is Raspbian OS. Just because all of those weak points of the surveillance system, an energy efficient portable system is proposed, that can take pictures when the burglary happens and send out an alert signal at the same time is much better than the current in use surveillance systems. It is simple to implement, small size portable stand-alone device with its own power source, energy capable with instantaneous alert, truly cheap for residential and personal use.

Suppose owner place a camera at one particular area which is to be monitored for security purpose, if user wants to go to remote location and still wants to monitor that same room for security, then he can make use of remote surveillance system by use of mobile through internet facility ${ }^{[1]}$. However, this can be used or can prove useful to a project targeting security setup which is limited to specific location to monitor properties and assets from a separate location.

\subsection{Why android?}

The main theme is the appearance of smart phone. As a smart phone appears in market, controller has tremendous change in design and installment. Smart phone has remarkable power in computation as well as a very convenient operation such as camera monitoring or wireless internet access by WIFI, with the help this powerful smart phone applications, the system becomes smarter and more intelligent ${ }^{[5]}$. Various application programs are open to market or developed. Android OS provides free SDK (Software Development Kit) which operates camera, speaker, audio, and Ethernet and it also has a merit of open source. Furthermore, in an integrated development environment called Eclipse an Emulator is provided.

Moreover, Internet of Things (loT) is an on going development of the internet by which everyday 'things' objects have communication capabilities which allow them to send and receive data. IoT based application can be used remotely to view the activity and get notification when motion is detected.

\section{LITERATURE SURVEY}

In literature ${ }^{[4]}$ an IOT based system and its advantages are explained which uses email notification and cloud to store data, literature ${ }^{[5]}$ discussed about remote image data transfer to android device with wireless LAN but with limited distance constrain.

The limitation of CCTV cameras is discussed in ${ }^{[6]}$ while the real time systems provide an immediate response for crime detection and the prevention. The main principle of network remote video surveillance system based on embedded system as discussed in literature ${ }^{[7]}$ is to set an embedded web server at the video surveillance terminal. The video signal should be 
digitized first, then compressed by the high compression chip and finally is sent to the built-in web server.

In literature ${ }^{[8]}$ power management is explained in sensor base system. The continuous monitoring of the videos is not required as discussed in ${ }^{[9]}$ where the video frames were compared with a template image. While comparing if any intrusion is detected then the authorized person is notified via GSM which include one extra hardware in system and make is costlier. ${ }^{[10]}$ talks about performance evolution of PIR sensor of deployment schemes, evaluated in a Java based simulation environment. They operate autonomously in unattended environments. And literature ${ }^{[11]}$ suggests system, captures video, shares among networked systems and also alerts the controlling person with short message service. This [12] considers visual transporting, storing, raising, using and connecting object with internet.

\section{PROPOSED WORK}

The aim is to make a smart surveillance system which can be monitored by owner remotely through android application. As it is connected with the system with IOT, system will send the push notification to android device when an intrusion is detected inside the room. It is required to develop and implement and affordable low cost web-camera based surveillance system for remote security monitoring. Authorized user can access to their monitoring system remotely via internet with the use a mobile phone and monitor the situation on application. This entire work is done on raspberry pi with Raspbian operating system ported on it.

Surveillance System consists of mainly two parts:

A. Hard-wired surveillance systems: These systems use wires to connect the cameras, motion detectors, power supply and LAN cable with the pi.

B. Remote Access Systems: These systems have the capability to monitor and control a security system from a location away from the surveillance area through android device.

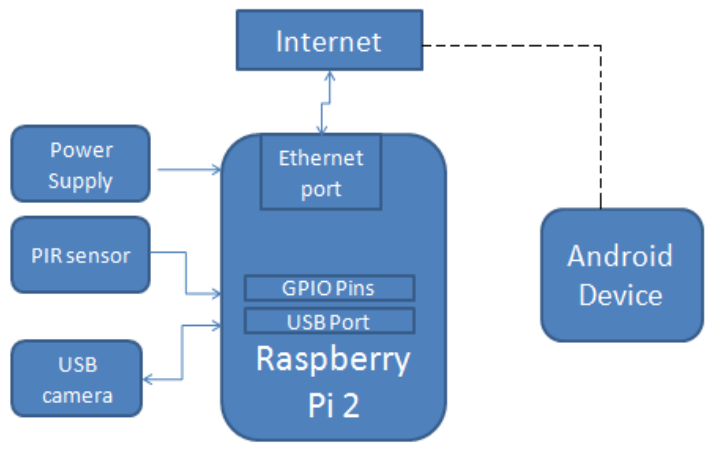

Fig 1: System Block Diagram

The block diagram shows that the project is divided into two sections. The raspberry pi will be somewhere at a remote place, in surveillance area, spying the activities. And the controlling of it will be done from any part of the world through android application. The system consists of an USB camera to capture the image from the crime scene.

\subsection{System Overview}

The functions of the various components are given below:
A. USB Camera: USB Camera captures the image and sends it to the USB port of the Raspberry Pi board. The camera model used here is USB Camera model 2.0.

B. Raspberry Pi: Raspberry pi is a small credit-card sized computer capable of performing various functionalities such as in surveillance systems, military applications, etc.

C. Android device: To view the captured images remotely and also receive the notification message.

D. PIR sensor: Detect the motion in surveillance area.

E. Pushetta Application: Sending notification with embedded system was a problem with few solutions, widespread was SMS but other side there are some issues of extra payment, limited number of data, etc. Pushetta provide push notification from cloud after getting registered on Pushetta website. After registering it requires

System requires $650 \mathrm{~mA} @ 5 \mathrm{v}$ power supply and internet. System works on Raspbian Os and image is captured through Opencv and programming is done in python scripting language. So for capturing images and interfacing, lots of packages need to be installed on Raspbian. Whenever PIR sensor detects any intrusion it will send positive signal to the raspberry pi GPIO pin. Pi senses the signal and send the output signal to USB camera for capturing image. Temporarily image is stored in raspberry SD card along with that it will send one notification message to user's android device through internet.

\subsection{Project Implementation}

The primary function of our project is:

Step 1: Setting up Raspberry Pi

After getting Raspberry Pi, SD Card is needed to be written with its desired OS. After getting ready with SD Card insert it into Raspberry Pi Micro USB Power Cable is attached with it along with Raspberry Pi with monitor through HDMI cable. Connect Keyboard \& Mouse with it. Also provide the DC power supply to Pi.

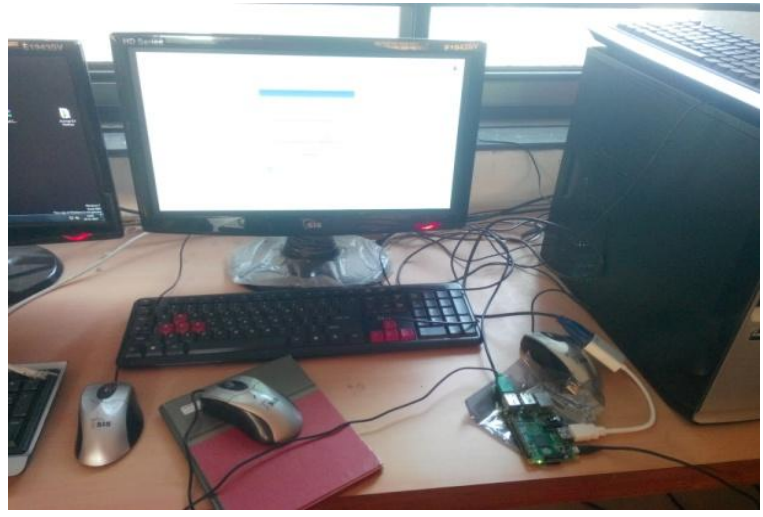

Fig 2: Raspberry Pi installation setup

Step 2: Operating raspberry pi without monitor display, keyboard and mouse.

Raspberry pi is needed to be attached with internet through RJ-45 cable, power supply and USB camera without need of any PC. Through PuTTy raspberry pi can be programmed and then works in standalone mode. PuTTY can be installed in Laptop and through IP address of raspberry pi it can access command window of pi. 


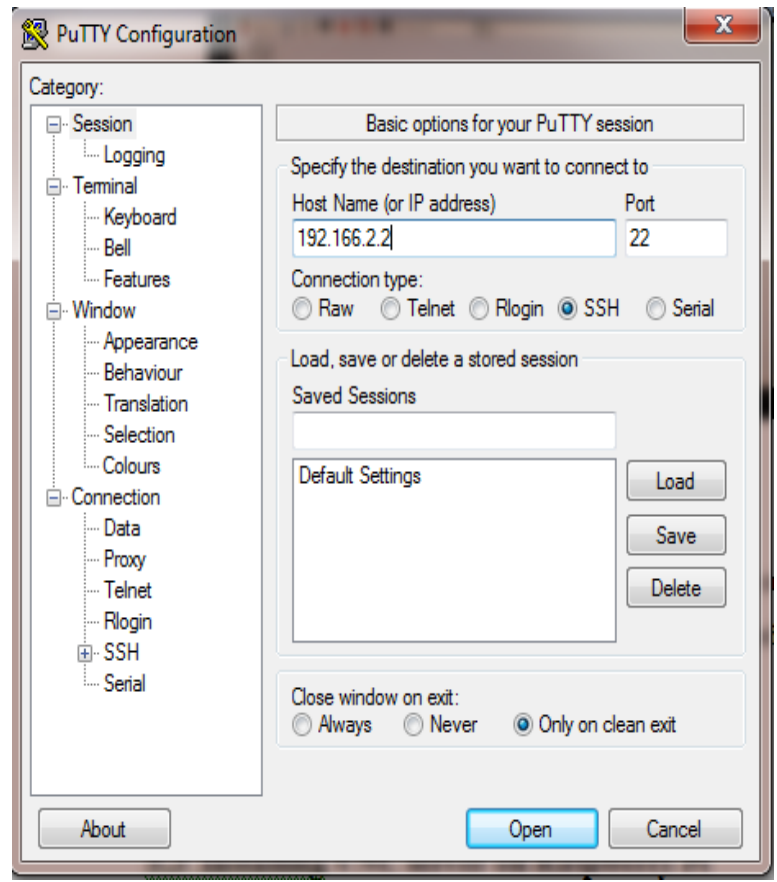

Fig 3: PuTTy configuration

Step 3: Installing VNC server on Raspberry Pi

To install VNC following command are used.

\$ sudo apt-get update

\$ sudo apt-get install tightvncserver

\section{Step 4: Client Side (Laptop)}

Downloading of VNC client and dynamically enter IP address of Raspberry Pi to operate pi through PC.As with SSH, since this is working over network, Pi could be situated anywhere, as long as it is connected to network.

Step 5: Running VNC server at start up in Raspberry Pi To ensure that VNC starts automatically, it enable the system working remotely with GUI display of Pi. Now it requires hardware connection of PI and sensor as shown in figure.

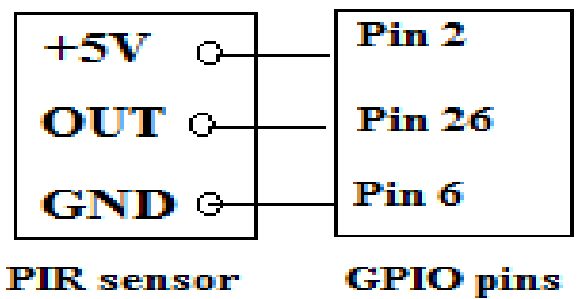

Fig 4: Interfacing of Raspberry pi with PIR sensor

Motion Detection: The python script matches the last frame and the present frame of the live video, if there is any difference then the motion flag is set, triggering all the events and snapshots generation will begin.

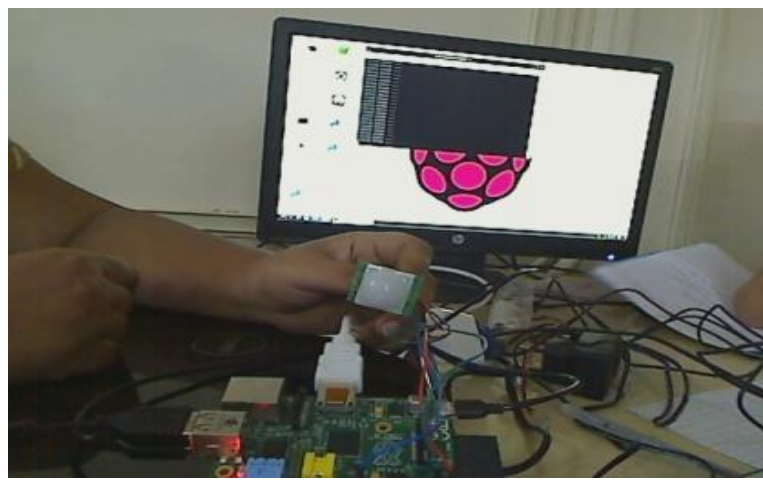

Fig 5: Image captured on motion detection

Sending Notification:

When the motion is detected, sensor data will trigger out and it will send one notification to android application used here is "Pushetta". It will that any intrusion is there inside the surveillance area and make the user aware of the situation. But before that one channel subscription is required and on movement it will send message to that particular subscribed channel.

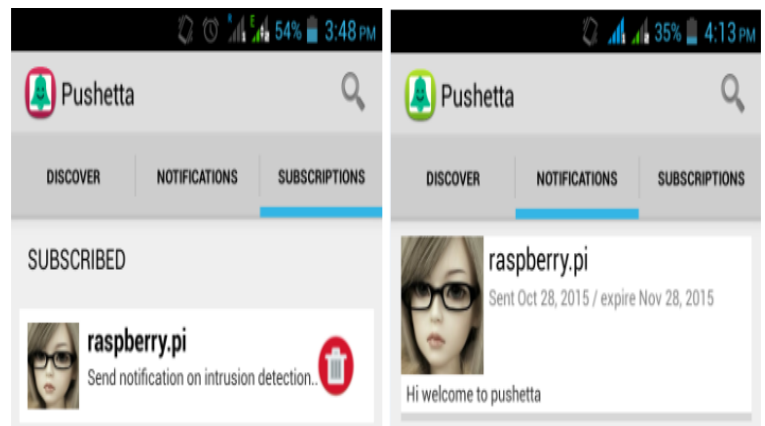

Fig 6: Screenshot of subscription and notification window of Pushetta

\section{FUTURE EXPANSION}

One application should be developed which include pushetta notification as well as the controlling power of raspberry pi from the window. User can also view captured image remotely on this application. Live video streaming can be added as per the user requirement. Power management should also be there and system went on sleep mode when it is no longer in active mode.

\section{CONCLUSION}

The smart surveillance system has been aimed to design in such a way that it can fulfil the needs of the user for particular surveillance area. It has countless applications and can be used in different environments and scenarios. For instance, at one scenario it can be used by any person working in industry to be aware of the activity being happened at their own working places, in their absence, while at another instance it can be used for spy purposes at bank lockers, storage houses. Another application is to provide information to the user about what is happening in surveillance area by notification.

\section{REFERENCES}

[1] Prof. Uma Nagaraj, Sayali Sonawane, Prachi Kalbhor,Sonali Diware, Shweta Iskande ,"Remote Surveillance System for Mobile Application ", International Journal of Engineering Research and Applications ,2012. 
[2] Sagar Joshi, Amit Joshi, Sanket Jabade, Ameya Jathar4 Electronics \& Telecommunication"M2M Communication Based Wireless SCADA forReal-Time Industrial Automation" International Journal of Research in Advent Technology, Vol.2, No.4, April 2014.

[3] V'ictor Bautista Saiz, Hospitalet de Llobregat Barcelona "GPU: Application for CCTV systems" International carnahan conference on security technology (ICCST), pp, 1-4, Rome, October 2014.

[4] Aamir Nizam Ansari \ Mohamed Sedkyl, Neelam Sharma2, Anurag Tyagil 1Faculty of Computing, Engineering and Sciences, Staffordshire University, Stoke-on-Trent, United Kingdom" An Internet of Things Approach for Motion Detection using Raspberry Pi" presented at the International Conference on Intelligent Computing and Internet of Things (IC1T),2015.

[5] Sung Wook Moon 1 , Young Jin Kim2, Ho Jun Myeong3, Chang Soo Kim4, Nam Ju Cha5, and Dong Hwan Kim 6 "Implementation of Smartphone Environment Remote Control and Monitoring System for Android Operating System-based Robot Platform”, 2011 8th International Conference on Ubiquitous Robots and Ambient Intelligence (URAI 2011), Songdo ConventiA, Incheon, Korea ,Nov. 2011

[6] X. Cai, F.H. Ali, And E. Stipidis, "Mpeg4 Over Local Area Mobile Surveillance System”, 2002.
[7] Linhua Ding, "Study of Embedded Linux Surveillance System Using TCP/IP Network", International Conference On Computer Design And Appliations (ICCDA 2010).

[8] Ying-Wen Bai, Zi-Li Xie and Zong-Han Li, "Design and Implementation of a Home Embedded Surveillance System with Ultra-Low Alert Power" IEEE Trans. Consumer Electronics, Vol.57. pp, 153-159, February 2011.

[9] Tasleem Mandrupkar,Manisha Kumari, Rupali Mane, "Smart Video Security Surveillance with Mobile Remote Control ", International Journal of Advanced Research in Computer Science and Software Engineering 2013.

[10] Z. Yu and W. Tie-Ning, 'Research on the Visualization of Equipment Support Based on the Technology of

[11] Internet of Things', 2012 Second International Conference on Instrumentation, Measurement, Computer, Communication and Control, 2012

[12] Serkan Akbas,Mehmet Akif Efe \& Suat Ozdemir "Performance Evaluation of PIR Sensor Deployment in Critical Area Surveillance Networks" , 2014 IEEE International Conference on Distributed 